\title{
Straw Man \\ 900-1000 GeV Crystal Extraction Test Beam for Fermilab Collider Operation
}

\author{
R. A. Carrigan, Jr. \\ Fermi National Accelerator Laboratory \\ P.O. Box 500, Batavia, Illinois 60510
}

October 1996 


\section{Disclaimer}

This report was prepared as an account of work sponsored by an agency of the United States Government. Neither the United States Government nor any agency thereof, nor any of their employees, makes any. warranty, express or implied, or assumes any legal liability or responsibility for the accuracy, completeness or usefulness of any information, apparatus, product or process disclosed, or represents that its use would not infringe privately owned rights. Reference herein to any specific commercial product, process or service by trade name, trademark, manufacturer or otherwise, does not necessarily constitute or imply its endorsement, recommendation or favoring by the United States Government or any agency thereof. The views and opinions of authors expressed herein do not necessarily state or reflect those of the United States Government or any agency thereof.

\section{Distribution}

Approved for public release: further dissemination unlimited. 
TM-1978

\title{
Straw Man \\ 900-1000 GeV Crystal Extraction Test Beam for Fermilab Collider Operation
}

\author{
R. Carrigan \\ Fermi National Accelerator Laboratory
}

October, 1996

\begin{abstract}
A design for a $900-1000 \mathrm{GeV}, 100 \mathrm{KHz}$ parasitic test beam for use during collider operations has been developed. The beam makes use of two bent crystals, one for extraction and the other one for redirecting the beam in to the present Switchyard beam system. The beam requires only a few modifications in the A0 area and largely uses existing devices. It should be straight-forward to modify one or two beam lines in the fixed target experimental areas to work above $800 \mathrm{GeV}$. Possibilities for improvements to the design to operate at higher fluxes are discussed.
\end{abstract}




\section{Introduction}

Recent work at Serpukhov ${ }^{1}, \mathrm{CERN}^{2}$, and Fermilab ${ }^{3}$ has demonstrated that bent crystals can be used effectively for extraction from accelerators. This technique has been used successfully at Serpukhov to extract a significant fraction of the circulating internal beam. The demonstration of multiple pass extraction at CERN has shown that this technique is even more successful than was originally anticipated. With multiple turns, the non-channeled beam passes through the crystal many times until it either interacts or channels and is extracted. Metaphorically, crystal extraction is a successful cross between a Maxwell demon and the Energizer Bunny. The angular divergence of the beam in the bend direction is small, on the order of two times the channeling critical angle.

The recent demonstration by E853 of a parasitic $120 \mathrm{Khz}$ extracted beam at C0 without undue background impact on the collider detectors is particularly relevant. During Run Ib, E853 operated with a bent crystal about five to seven sigma from the circulating proton beam. The system was able to extract more than $100 \mathrm{KHz}$ without tripping the CDF and D0 loss monitors. Parenthetically it should be noted that the collider loss monitor trip points were set quite conservatively and there was little head room for losses.

While a $900-1000 \mathrm{GeV}$ beam to the fixed target areas would have 12 to $25 \%$ higher energy than is currently available, there is probably no significant new physics to be done because the parasitic beam intensity would be rather low. On the other hand, the beam could be quite useful for tests both for future Fermilab projects and for LHC development. The spill structure would be relatively uniform and the beam-up time would correspond to the "on" time for the collider detectors. In other words, there would not be the duty factor for magnet ramping for the fixed target program. With the Switchyard the beam could be switched to several different experimental areas. The beam would not be split because the intensity is already low. This fact also lessens the problems in operating the Switchyard above $800 \mathrm{GeV}$.

The following sections describe the basic design of the crystal extraction straw man beam, the expected intensity, the spill structure, required goniometers and crystals, the beam installation including interferences that must be considered, instrumentation and tuning, operation of the Switchyard at $1000 \mathrm{GeV}$, and possible improvements.

\section{Basic Design}

For parasitic operation where the crystal scrapes the portion of the circulating beam at a large distance from the central core, the crystal will operate much like the E853 crystal. The principal difference is that a much larger bend angle is required to avoid obstructions at AO and to mate to the Switchyard beam line.

In operation the crystal is moved toward the beam until it becomes the principal scraper in the Tevatron. Thus all the halo beam that arises from emittance growth strikes the crystal. At high intensity most of the emittance growth is due to beam-beam interactions. 
A logical location for the bent crystal is just after the last $\mathrm{A} 0$ proton kicker magnet (see Figure 1). This magnet chain normally kicks the beam into the $10 \mathrm{~m}$ long AO abort absorber ${ }^{4}$. The absorber is surrounded by cooled Tevatron magnet laminations. These are loosely surrounded by a steel shell. A minimum bend of $13.4 \mathrm{mrad}$ is required to just miss the 4.75 inch half width of the laminations.

A reasonable place to reconnect with the switchyard beam line is at PV92 (near the gate that separates Enclosure B from A0). Most of the elements of PSEP, the Proton Area electrostatic separator, just upstream of PV92 would have to removed from the beam. They are easy to remove and not required for collider operation. There is room to move them to the west side of the A0 enclosure. A horizontal bend of $15.7 \mathrm{mrad}$ for the extraction crystal is required to cross the switchyard beam at PV92.

A vertical bend of 4.7 mrad is also needed to cross at PV92. Both bends can be provided by tilting the crystal just as is done with the skew dipole chain currently used for fixed target operations. The combined bend (horizontal and vertical) that is required is $16.4 \mathrm{mrad}$. The extraction and transmission efficiency of the extraction crystal is about $9 \%$.

The beam must be redirected on to the switchyard line at PV92. This requires a horizontal bend of $7.17 \mathrm{mrad}$ and a downward vertical bend of $2.17 \mathrm{mrad}$ for a total bend of 7.49 mrad. A simple way to do this is to use a second "redirection" crystal. (Multiple crystal geometries have already been used several times at Serpukhov".) Because the angular divergence of the beam out of the extraction crystal is only two times the critical angle for channeling (about ten microradians total), the beam spot size in the bending direction $300 \mathrm{ft}$ downstream is only $1 \mathrm{~mm}$. Thus a $2 \mathrm{~mm}$ thick crystal would leave margin for survey error and other uncertainties.

For this situation the transmission through the redirection crystal is on the order of $40 \%$. An alternative approach would be to introduce 4-5 EPB dipoles or several Tevatron dipoles.

One important difference between $\mathrm{A} 0$ and the $\mathrm{C} 0$ straight section where $\mathrm{E} 853$ was located is that the proton beam was outside the ring at $\mathrm{C} 0$ but is inside the ring at $\mathrm{A} 0$. Most of the crystal bend is to the outside and the sagitta of the crystal is significant compared to the various step sizes in the problem. As noted in the introduction, much of the beam that passes through the crystal first multiply scatters for several turns before it is channeled. Understanding whether the crystal could be practically moved in from the side requires simulation studies. A different option more in line with E853 operation would be to move the crystal in from above (Figure 2). This is the so-called Murphy-Newberger geometry where the beam comes in between the crystal planes rather than crossing the planes. Even that may have complications with the tilted crystal. For example, the beam would scrape in both $\mathrm{x}$ and $\mathrm{y}$. One way to circumvent that would be to bevel the crystal. Understanding the best approach to handling the tilted geometry requires further study. However, this should not be a major problem.

A second complication for crystal operation is that the crystal must be aligned to the 
channeling angle in a coordinate system that is tilted. This could be handled by either tilting the present E853 goniometer or doing this by a software transformation. Both are feasible.

\section{Expected Intensity}

The crystal extracted beam intensity is directly proportional to the circulating proton beam loss rate. Increasing the loss rate decreases the luminosity lifetime and increases the backgrounds at the collider detectors. There is a natural loss rate that occurs because of beam-beam interactions, gas scattering, and any other source of beam growth. Collider interactions are dominant for present and future luminosities at the Tevatron. These natural losses must be controlled by systematically scraping the circulating beams in the Tevatron. This is done for protons with scrapers at D17 (primary horizontal and vertical), F49 (secondary vertical), and A0 (secondary horizontal).

In spite of the scraping, there are still losses at the collider detectors. Characteristically the beam is scraped until the detector losses are below some trip point that provides adequate protection and clean operation for the detectors but does not cut the luminosity lifetime. The establishment of these trip points is not so much high science as a compromise between conflicting requirements.

The beam rate down the switchyard line is then a function of the beam loss in the accelerator, the fraction of the beam losses that strike the crystal, the overall extraction and deflection efficiency of the crystal system, and the maximum allowed loss rate at the detectors. For a typical E853 situation the CDF LOSTP limit was $<5 \mathrm{KHz}$ with a typical loss rate of 3.5 $\mathrm{KHz}$ without the crystal, the D0 limit was DOHLP $<1 \mathrm{KHz}$ with a typical loss rate quite close to that, 6 bunches were colliding, the circulating proton current was $10^{12}$, the proton lifetime was 75 hours, the luminosity lifetime was 15 hours, and the CDF luminosity was $0.5^{*} 10^{31}$. For the E853 C0 crystal D0 was directly downstream with no scrapers in between. Perhaps for this reason, losses at D0 usually turned out to be the limitation. For A0 the situation is reversed (CDF is closer to the crystal than D0) and all of the scrapers are after the two detectors.

Under good conditions in E853 where the crystal was the principal aperture the fraction of the beam scraped on the crystal was on the order of $25 \%$ based on measurements of the lifetime with the crystal in and out of the beam. A good extracted rate within the CDF and D0 limits was $100 \mathrm{KHz}$ based on end-of-store results. The extraction efficiency was $\mathrm{O}(20 \%)$ so that $500 \mathrm{KHz}$ was lost on the crystal. The proton beam lifetime indicates that the average loss rate around the machine was $4000 \mathrm{KHz}$. The inconsistencies in these numbers reflect a variety of different conditions and also the fact that they express order of magnitudes.

To estimate the beam rate down the switchyard line after the Main Injector is in operation one has to work forward from the E853 data by multiplying by 6 to take into account the increase to 36 bunches. In addition it is assumed that the background rates at the detectors per bunch are held constant so that the background per second can increase by a factor of 6 . The crystal extraction efficiency has to be unfolded from the E853 results and extrapolated to the A0 
crystal geometry. This can be approximated using the results of the Biryukov simulation ${ }^{6}$ for E853 and other models of the channeling process ${ }^{7}$.

If the beam emittance is substantially greater than the crystal acceptance the transmission efficiency of a bent crystal septum in an external beam can be approximated by ${ }^{8}$ :

$$
E=E_{c}\left(\frac{\phi_{b}^{50}}{\Phi}\right)\left(1-\frac{p}{3 R_{m}}\right) e^{\left(-5 p_{0} / \lambda_{0} P\right)}
$$

In the formula $\phi_{b}^{50}$ is the phase space acceptance of the bent crystal (proportional to the channeling critical angle in this case), $\Phi$ is the $50 \%$ phase space emittance of the particle beam for the crystal bending direction, $\mathrm{E}_{\mathrm{c}}$ is the surface acceptance of the crystal, $\mathrm{R}_{\mathrm{m}}$ is the minimum radius of bend along the crystal in $\mathrm{cm}, \mathrm{p}$ is the beam momentum in GeV/c, $\mathrm{s}$ is the length of the crystal, and $\lambda_{0}$ is the bent crystal dechanneling length at $\mathrm{p}_{0}$ (taken as $8.25 \mathrm{~cm}$ at $100 \mathrm{GeV} / \mathrm{c}$ ). To account for the decreased channeling length in a bent crystal ${ }^{1}$ the dechanneling length for the straight crystal case is multiplied by $\left(1-\mathrm{p} / 3 \mathrm{R}_{\mathrm{m}}\right)^{2}$.

For accelerator extraction, a circulating particle that is not channeled often remains in the accelerator and is channeled on a later pass. This multiple pass extraction ${ }^{9,10.11}$ leads to an effective higher overall efficiency for the channeling extraction process when the beam divergence is greater than the channeling angle. For accelerator extraction the first two terms in (1) should be replaced by an extraction efficiency, $E_{e}$, so that the overall extraction and bending efficiency for the accelerator case is:

$$
E_{a}=E_{e}\left(1-\frac{p}{3 R_{m}}\right) e^{\left(-s p_{0} / \lambda_{0} p\right)}
$$

For maximum transmission efficiency in an external beam, the crystal phase space acceptance should be as close to the beam emittance as possible or even larger. This is less of a factor in an extraction crystal because of the effect of multiple passes. Understanding of multiple pass extraction efficiency is still in a nascent stage. The recent CERN measurements at $120 \mathrm{GeV}$ in an accelerator lattice configuration not specifically optimized for channeling extraction give $E_{a}$ in the range of $10-15 \%$. Fermilab has observed extraction at $900 \mathrm{GeV}$ but no efficiency measurements have been reported yet. The simulations by Biryukov for $900 \mathrm{GeV}$ and 7.7 $\mathrm{TeV}^{12}$ give values of $\mathrm{E}_{\mathrm{a}}$ as high as 0.7 .

The Biryukov simulation models the specific E853 conditions ( $900 \mathrm{GeV}, 640 \mu \mathrm{rad}$ ) but also studies the behavior of the extraction efficiency as a function of crystal length. A simple model has been devised to extrapolate this simulation to other bend angles. The amount of the beam that is extracted is the sum over all the passes through the crystal. On the jth pass the amount of the remaining beam that is extracted is given by the ratio of the channeling critical angle divided by the angular half width of the beam for that pass $\left(\psi_{\mathrm{c}} / \Theta_{\mathrm{j}}\right)$. The amount that is lost is the ratio of the crystal length (or, in some cases, the length of the crystal the beam passes through) divided by the nuclear interaction length $\left(L / L_{n}\right)$. The portion of the beam that remains 
in the accelerator after each turn is:

$$
\left(1-\frac{\Psi_{c}}{\Theta_{j}}-\frac{L}{L_{n}}\right)
$$

The size of $\Theta_{j}$ is due to the angular distribution of the beam and multiple scattering from the earlier passes. Typically the beam angular distribution is $\mathrm{O}(10 \mu \mathrm{rad})$. Biryukov notes that one yardstick of the multiple scattering can be obtained by substituting the nuclear interaction length in the normal multiple scattering formulation. This gives $30 \mu \mathrm{rad}$ for this case. To arrive at an easily summed ansatz we assume that $\Theta_{j}$ is a constant $\Theta_{\mathrm{III}}$. The extraction efficiency is then the sum of the fraction extracted on each turn $\left(\psi_{c} / \Theta_{m}\right)$ times the proportion remaining at that turn (eq. $3)$. In that case the extraction efficiency is:

$$
E_{\theta}=\frac{1}{\left(1+\frac{\Theta_{m}}{\Psi_{c}} \frac{L}{L_{n}}\right)}
$$

$\Theta_{m}$ is then fitted to conform to the Biryukov distribution at large L. In Figure 3 the results of the fitted ansatz (solid line) are compared to the Biryukov distribution (dashed line) with $\Theta_{\mathrm{m}}=$ $38 \mu \mathrm{rad}, 25 \%$ larger than the Biryukov many-pass multiple scattering angle. The ansatz appears to underestimate extraction efficiencies for short crystals but gives a good approximation for longer ones.

Equations (2) and (4) have been used to optimize the crystal lengths and determine the efficiency for the extraction and redirection crystals. The efficiency curve is shallow around the maximum so that there can be quite a bit of latitude in choosing the length of the crystals. For $12.5 \mathrm{~cm}$ long crystals $\mathrm{E}=9 \%$ for the extraction crystal while $\mathrm{E}=40 \%$ for the redirection crystal. This gives an overall transmission for the beam of $3.6 \%$. Assuming $6 \mathrm{MHz}$ is lost on the crystal under Main Injector conditions the beam transmitted down the Switchyard line will be $100 \mathrm{KHz}$.

\section{Spill Structure}

Several elements enter into the character of the spill structure for this beam. One is the RF bucket structure. A second is the beam intensity over the lifetime of a store. A third relates to fluctuations due to small orbit changes in the accelerator.

With 36 bunch operation, the Tevatron bucket frequency is $1.7 \mathrm{MHz}$. For a $100 \mathrm{KHz}$ beam rate most buckets will be unoccupied. Even at $1 \mathrm{MHz}$ there will be relatively little multiple occupancy in a bucket. Thus ordinary scintillation counting of the beam is no problem.

For a typical twenty hour store in the Tevatron, the luminosity decreases several fold. Since beam-beam interactions are the major driving factor moving beam out to the crystal the expected beam should also drop. However, detector background rates should also drop (during E853 CDF dropped but D0 increased with time into the store) so in principal it might be possible 
to scrape harder by moving the crystal in during the store. As a result it might be possible to keep the intensity relatively constant over the cycle. (Note again that the $100 \mathrm{KHz}$ estimate above is based on E853 end-of-store data.)

During E853 operations there were indications of beam wandering in the Tevatron by up to 100 microns. Some of this could be attributed to the presence of the Main Ring which will not be operating during the Main Injector epoch. A 100 micron beam fluctuation can produce a substantial modulation of the spill intensity. Understanding of this type of problem in the Tevatron is evolving. With time and experience more insight will be gained. This could also have other beneficial effects for Tevatron operation.

One frequently expressed coneremout crystal extraction is the possibility of radiation damage to the crystal. There was no evidence of damage at all in the 70 hours of operation for E853. However an extraction crystal would have to last about 100 times longer. Radiation damage of crystals used for high energy channeling has now been studied in some detail. Measurable damage ${ }^{13}$ with an effect on channeling occurs at a rate on the order of $10^{20} / \mathrm{cm}^{2}$. Assuming the active region of the crystal is 10 microns wide and $0.6 \mathrm{~mm}$ high, an extraction crystal should be able to handle on the order of $10^{16}$ particles. At $1 \mathrm{MHz}$ this would give a lifetime of the order of a thousand years, well above what is probably required.

\section{Goniometers and Crystals}

As noted above, $12.5 \mathrm{~cm}$ long crystals would optimize the transmission. These are three times longer than the E853 crystal and will probably require obtaining a 6 inch diameter silicon boule. These are available but expensive (thousands of dollars). Serpukhov has already used several longer crystals ${ }^{1}$. The A0 crystals would be about $1 \mathrm{~cm}$ wide for ease of fabrication and alignment and $2 \mathrm{~mm}$ thick. This is $33 \%$ thinner than the crystal used in E853 but should provide adequate coverage of both the Tevatron and the external beam. Going to a thinner crystal allows a little more safety factor for the larger bend.

The crystals have to be cut with a (110) or (111) plane of the crystal aligned within 100$200 \mu \mathrm{rad}$ of the major face of the crystal and with the beam-facing surface optically flat. Crystal cutting and preparation could be handled in the same manner as the E853 crystals. They were prepared by a firm in St. Petersburg, Russia working in collaboration with the Petersburg Nuclear Physics Institute. The silicon boule for E853 was obtained from Wacker Corporation in Germany. A $4 \mathrm{~cm}$ long section of that $7.5 \mathrm{~cm}$ diameter boule is still available but it cannot provide a long enough crystal.

The goniometer used for E853 performed satisfactorily. It is a $1 \mathrm{~m}$ section of Tevatronquality bakeable vacuum pipe outfitted with bellows at both ends. Four degrees of freedom are provided by $\mathrm{x}$ and $\mathrm{y}$ motions at both ends. These are moved by stepping motors controlled by ACNET. The crystal is located over the upstream $x$ and $y$ motions so that crystal angles are controlled by the downstream motions. (Crystal positions require controlling two motions.) The tilted geometry could be accommodated by either tilting the entire goniometer or through 
software. For historical reasons one of the four stepping motors has a minimum step size of 12.5 microns while the other three have a 2.5 micron step size. It would be helpful to use 2.5 micron steppers for all four motions to improve the crystal horizontal positioning which is now rather coarse. The E853 goniometer could accommodate up to a 3.75 in long crystal $(9.5 \mathrm{~cm})$. Going to this shorter length would reduce the efficiency by $\mathrm{O}(10 \%)$

The goniometer for the redirection crystal would not have to be so sophisticated. For example it would not have to be bakeable so the channeling angle alignment motion could be brought in through a good O-ring. The linear motions could be provided by duplicating one end of the E853 goniometer. Only one angular degree of freedom and perhaps only one linear motion are needed. Alternatively, if design effort was the limiting resource, the E853 system could just be duplicated.

Although the bend angles are larger, the E853 four point bender design can readily be adapted to the longer crystals.

\section{Beam Installation}

Handling the vacuum line from the extraction crystal to the A0 dump would be straightforward. This is a warm section of the Tevatron vacuum system. The line would be enlarged to a 6 inch pipe displaced to the outside of the ring for $20 \mathrm{ft}$ after the goniometer. At that point a bellows and a 2 inch diameter pipe would mate into a pipe threading through the abort shield.

A0 Beam absorber: The A0 absorber consists of cooled Tevatron magnet laminations with some plumbing along the side for the cooling. This is surrounded by a 4 in thick steel shield spaced about 6 inches away from the Tevatron core assembly (see the photographs in Figure 4). There is room for the beam to pass between the steel shield and the laminations if the plumbing is rerouted and some auxiliary shielding is moved. A straightforward modification of the manifold should work for the plumbing. A small steel plate is visible in the photograph. This might have to be cut away but there already appears to be a clear hole through to the end of the absorber. Some of the side support jacks on the absorber would have to be modified.

There are a series of potential interferences along the path of the extracted beam. Although there is vacuum outrigging on the skew dipoles, it will not be any concern since the skew dipoles are not in place during collider operations. Just after the skew dipoles about $90 \mathrm{ft}$ from $\mathrm{A} 0$ there is a vacuum pipe that curls outside of the present extraction line (Figure 5). There is also a $110 \mathrm{~V}$ unistrut power post nearby. Both may have to be rerouted for the crystal line. An aluminum box $110 \mathrm{ft}$ from A0 that holds the XSEM monitor (Figure 6) in the present line is well inside the path of the new line.

A substantial Tevatron current lead box comes down from the ceiling on the west side of V90 about $120 \mathrm{ft}$ from A0 and goes into a cryogenic fixture (Figure 7). The beam line may be inside this fixture but careful measurements are needed to check the clearance. Rerouting the 
leads would require a new fixture.

There is sufficient clearance around the existing $\mathrm{H} 90$ trim for the beam (Figure 8). A loss monitor on the side of the second trim dipole might have to be relocated. There is a substantial vacuum pump system about $170 \mathrm{ft}$ from A0 associated with PSEP, the proton area electrostatic splitter (Figure 9). Presumably this can be removed or relocated when PSEP is not needed during collider operations. Vacuum connections to the exiting switchyard line in the PSEP region would be straight-forward.

\section{Instrumentation and Tuning}

For an intensity of $\mathrm{O}(1 \mathrm{MHz})$ spread over 36 bunches there is not a major problem with using scintillation counters to monitor the beam. A system similar to the one employed for E853 could be used. The E853 system consisted of two pairs of scintillators separated by $40 \mathrm{~m}$ along with 1/32 inch thick moveable finger counters to measure the $\mathrm{x}$ and $\mathrm{y}$ distribution of the beam. An interaction counter system also looked at the crystal at 90 degrees to the beam. The location of the pairs of counters along the beam represents a compromise between wanting them close to the crystal so alignment problems are minimized and wanting them further down the line to minimize accelerator background.

The extracted beam is tuned by carefully moving the crystal in until it is a principal loss aperture. The extraction crystal channeling angle is then tuned by scanning the crystal plane angle and looking for a rise of the beam signal in the downstream scintillators and a drop in the interaction counter rate. Because of multiple pass effects the angular width of the channeling alignment curve is 50 to $100 \mu \mathrm{rad}$ wide so that the alignment curve can be taken in fairly large steps. One complication of E853 operations, gating out the Main Ring, will no longer be present.

After aligning the extraction crystal channeling angle the beam must be tuned so that it hits the face of the redirection crystal. The most critical adjustment is probably to compensate any mis-setting in the extraction crystal bend angle. A 1 mrad error in setting the bend results in missing the crystal face by about $10 \mathrm{~cm}$. One way to compensate this would be to make the crystal bend adjustable. This was done for the crystals used in E761, where $\Sigma$ magnetic moment precession with a crystal was demonstrated ${ }^{14}$. It is a complication, particularly in the high vacuum environment of the Tevatron. A second way to handle this would be to add a dipole to the extraction line. A $10 \mathrm{ft}$ EPB dipole, such as one of the $\mathrm{H} 90$ elements, can give a $1.5 \mathrm{mrad}$ deflection. An $\mathrm{H} 90$ element would be relatively easy to move, cable, power, and instrument. A small scintillation counter with the same area as the face of the redirection crystal could simplify this adjustment.

The channeling angle alignment of the redirection crystal would be handled in the same way. Another pair of scintillators should suffice. Existing magnets in the switchyard line could be used to adjust any mis-setting of the bend angle. 


\section{Operation of the Switchyard at $1000 \mathrm{GeV}$}

There are at least three ways where limitations could appear in trying to operate parts of the Switchyard at $1000 \mathrm{GeV}$ :

1) Bends-one hope is that there is headroom in some of the existing bending magnet chains. Another possibility is to add additional bending magnets in one or two beam lines. A third crystal could be used but that would further reduce the beam intensity.

2) Focusing-here the small angular divergence of the crystal beam may help. Detailed investigations of the beam optics are required.

3) Beam splitters- electrostatic septa will not be necessary for the crystal beam since the beam intensity is small and only one line could be fed at a time.

Presumably each beam line has different constraints for bending and focusing. Some lines may be more adaptable than others.

A different problem is monitoring the crystal beam. Most of the present instrumentation will probably not work with a $100 \mathrm{KHz}-1 \mathrm{MHz}$ beam since the beam intensity is down by factors of $10^{3}$ to $10^{6}$ from normal external beams. This problem would have to be addressed. Scintillators could provide relatively simple solutions.

The fact that modifications to both bends and instrumentation may be required suggests that it may be useful to focus on operating only a few beam lines rather than the entire Switchyard.

Another complication for Main Injector era Switchyard operation is that the $120 \mathrm{GeV}$ Main Injector operation will be used to feed several of the external beams (KAMI, Meson Area). At this stage there is not a formal planning document for the Switchyard beams. However a separate beam is planned out to the Road D region. Presumably this would be on the west side

of the A0 enclosure so that there would still be space for a crystal extracted line near the present line.

\section{Possible Improvements}

One possibility would be to replace the redirection crystal by $40 \mathrm{ft}$ of bending magnet. There is adequate room to install such a string in place of PSEP. Supplies and leads for V90, Q90, and V91 might be used. This would increase the beam intensity by a factor of 2.5 .

A second possibility is to use a germanium crystal (germanium has a larger critical angle) with a smaller bending angle to feed a septum magnet chain such as the Lambertson string that will no longer be needed for collider operation. This would probably require modifying the A0 
dump. This improvement could result in a gain of 2-4. The combination of this and replacing the redirection crystal could result in a flux gain of ten.

A third possibility is to employ a pre-scatterer such as the one-quarter channeling wavelength crystal proposed by Tsyganov and Taratin ${ }^{15}$ or an amorphous target such as a 100 $\mu$ carbon filament proposed by Asseev and his colleagues ${ }^{16}$. Pre-scatterers may raise the extraction efficiency. In this case the gain would probably be less than a factor of two. Most of the beam loss in the extraction crystal is due to the large bend required to clear the beam dump and the magnets about $200 \mathrm{ft}$ from $\mathrm{A} 0$.

A fourth possibility is to cool the crystal ${ }^{17}$. This would increase the dechanneling length and generally improve the extraction efficiency, perhaps up to a factor of two. In view of the fact that the goniometer is near the cold portion of the Tevatron vacuum system this might not be too difficult. Beam heating of the crystal is probably not a significant thermal load.

\section{Summary}

A two crystal system could be used to extract a $900-1000 \mathrm{GeV}$ beam from the Tevatron during Main Injector colliding beam operations. Beam intensities on the order of $100 \mathrm{KHz}$ appear quite practical with no significant impact on the collider detectors. Indeed, in the end the crystal system might provide somewhat cleaner scraping than the existing system and lessen background. The crystal geometries that are proposed use techniques proven in the E853 tests and elsewhere. Much of the equipment already exists. Costs including rigging might be between $\$ 50,000$ and $\$ 100,000$.

It would appear that such a system could have important use as a test beam both for collider detector upgrades and LHC detectors.

The author would like to thank C. Moore and T. Murphy for many helpful discussions. 


\section{References}

1. See, for example, V. M. Biryukov, V. I. Kotov, and Yu. A. Chesnokov, PhysicsUspekhi 37, 937 (1994) and references cited therein.

2. H. Akbari, et al., Physics Letters, B313, 491 (1993).

3. T. Murphy, et al., Nucl. Instr. and Meth., to be published.

4. "The A0 Abort System for the Tevatron Upgrade", C. Crawford, Fermilab TM-1564 (1989).

5. N. A. Galyaev, et al., 90-19 (1990). V. I. Baranov, V. M. Biryukov, A. P. Burgorsky, Yu. A. Chesnokov, V. I. Kotov, M. V. Tarakanov, V. I. Terekhov, S. V. Tsarik, O. L. Fedin, M. A. Gordeeva, M. P. Gur'yev, Yu. P. Platonov, and A. I. Smirnov, Nucl. Instr. and Meth. B95, 449 (1995).

6. V. Biryukov, Phys. Rev. E52, 6818 (1995).

7. R. Carrigan, p. 339 in Relativistic Channeling, eds. R. Carrigan and J. Ellison, Plenum (1987), New York. R. Carrigan, Fermilab 95-366 (1995), to be published in NIMB.

8. S. I. Baker, R. A. Carrigan, Jr., R. L. Dixon, H. C. Fenker, R. J. Stefanski, J. S. Forster, R. I. Wijayawardana, and S. Reucroft, Nucl. Instr. and Meth. A248, 301 (1986).

9. V. Biryukov, Nucl. Instr. and Meth. B53, 202 (1991).

10. A. Taratin, S. Vorobiev, M. Bavizhev, and I. Yazynin, Nucl. Instr. and Meth. B58, 103 (1991).

11. X. Altuna et al., Phys. Lett. B357, 671 (1995).

12. V. Biryukov, Phys. Rev. Lett. 74, 2471 (1995).

13. S. I. Baker, R. A. Carrigan, Jr., V. R. Cupps II, J. S. Forster, W. M. Gibson, and C. R. Sun, Nucl. Instr. and Meth. B90, 119 (1994).

14. V. V. Baublis, et al., Nucl. Instr. and Meth. B90, 150 (1994).

15. E. Tsyganov and A. Taratin, Nucl. Instr. and Meth. A363, 511 (1995).

16. A. A. Asseev, E. A. Myae, S. V. Sokolov, and Yu. S. Fedotov, Nucl. Instr. and Meth. A324 31 (1993). 
17. J. S. Forster, H. Hatton, R. J. Toone, G. Este, S. I. Baker, R. A. Carrigan, Jr., W. M. Gibson, R. L. Wijayawardana, J. A. Ellison. L. Emma-Wori, B. O. Kolbesen, Nuclear Physics B318, 301 (1989). 


\section{Figure Captions:}

1. Beam layout for crystal extraction beam at A0. Note the absorber near A0, clearances near XSEM, H90, and V91 at about $150 \mathrm{ft}$ after A0, and the location of PSEP. The insets at the center show the decomposition of the crystal bend vectors. Scale $\mathrm{x}: \mathrm{y}$ is 10:1.

2. Beam geometry at the extraction point. The proton beam is shown at $\mathrm{x}=-3.13 \mathrm{~mm}, \mathrm{y}$ $=-1.03 \mathrm{~mm}$. Views are schematic. The plan view is distorted. The crystal is shown 5 $\sigma$ from the beam. An arrow in the beam view indicates the direction of bend. The right hand side is inside the ring.

3. Extraction efficiency as a function of crystal length $\mathrm{L}$ in $\mathrm{cm}$ for $\mathrm{E} 853$ conditions. The Biryukov simulation is shown as a dashed line while the simple ansatz fitted to the Biryukov simulation at large $\mathrm{L}$ is shown as a solid line.

4. Pictures of the A0 absorber. Note the surrounding blue shield, plumbing for cooling, and the floor supports.

5. Vertical vacuum piping $90 \mathrm{ft}$ after $\mathrm{A} 0$ that circles the external beam line. A vertical 110 $\mathrm{V}$ outlet post is just visible behind the vacuum pipe.

6. Aluminum box at $110 \mathrm{ft}$ for the XSEM monitor.

7. Two views of Tevatron current lead near $120 \mathrm{ft}$.

8. $\mathrm{H} 90$ trim magnet near $135 \mathrm{ft}$. Note the loss monitor on the side of the second trim magnet.

9. PSEP vacuum pump at $170 \mathrm{ft}$. This would not need to be present during collider operations. 